\title{
On finite pattern-free sets of integers
}

\author{
by \\ KARL Dilcher (Halifax) and \\ Lutz G. Lucht (Clausthal-Zellerfeld)
}

1. Introduction. Many classical problems of combinatorial number theory concern extremal sets $A$ of positive integers that do not contain certain patterns. A general definition was given in [6]: Let $\sigma$ be a nonempty family of finite nonempty subsets of $\mathbb{N}$. A set $A$ of positive integers is called $\sigma$-free if no subset of $A$ belongs to $\sigma$. We call the system $\sigma$-invariant if it is both homogeneous, i.e., $k \mathcal{S}:=\{k s: s \in \mathcal{S}\} \in \sigma$ for all $k \in \mathbb{N}$ and $\mathcal{S} \in \sigma$, and translation-invariant, i.e., $k+\mathcal{S}:=\{k+s: s \in \mathcal{S}\} \in \sigma$ for all $k \in \mathbb{N}$ and $\mathcal{S} \in \sigma$.

In many cases the forbidden patterns are the solutions $\mathcal{S}:=\left\{x_{1}, \ldots, x_{k}\right\}$ $\subset \mathbb{N}$ to linear equations of the form $a_{1} x_{1}+\cdots+a_{k} x_{k}=b$ with fixed integer coefficients $a_{1}, \ldots, a_{k}, b$. Invariance then means that $a_{1}+\cdots+a_{k}=b=0$, and in order to avoid trivialities, it is understood that $x_{1}, \ldots, x_{k}$ are distinct. A typical example is the system $\sigma$ of sets $\{x, y, z\} \subset \mathbb{N}$ such that $x+y=2 z$, $x \neq y$. Here the $\sigma$-free sets $A$ of natural numbers are those that do not contain arithmetic progressions of length 3 .

In the following we only consider noninvariant homogeneous systems $\sigma$ consisting of the solutions $\{x, y, z\} \subset \mathbb{N}$ to an equation of the form

$$
a x+b y=c z
$$

with given coefficients $a, b, c \in \mathbb{N}, \operatorname{gcd}(a, b)=1, a \leq b$, and $c$ sufficiently large. For short we say that $\sigma$ is defined by (1.1). Among various possible density notions we choose as the most natural one in this setting the upper maximal density bound

$$
\bar{D}(\sigma)=\lim \sup \left(\frac{f(\sigma, n)}{n}\right),
$$

where $f(\sigma, n)=\max \{|A|: A \subseteq\{1, \ldots, n\} \sigma$-free $\}$ denotes the largest possi-

2000 Mathematics Subject Classification: Primary 11B75; Secondary 05B10, 05D05.

This research project was supported by the Natural Sciences and Engineering Research Council of Canada. 
ble cardinality of a $\sigma$-free subset of $\{1, \ldots, n\}$ (compare with Ruzsa [10], [11], Chung and Goldwasser [4], and Chung, Erdôs and Graham [2]).

TheOREM 1. Let the system $\sigma$ be defined by (1.1) with $a=1$ and positive integers $b, c$ satisfying $c \geq 2(a+b)^{2}-\operatorname{gcd}(a+b, c)$. Then, with $q=(a+b) / c$, $\alpha=a q / c$ and $\beta=b q / c$, we have

$$
\bar{D}(\sigma)= \begin{cases}(1-q) \frac{1-\alpha^{2}}{1-\alpha-\alpha^{2}} & \text { for } b=1, \\ (1-q) \frac{1-\beta+\alpha}{1-\beta} & \text { for } b>1 .\end{cases}
$$

To a certain extent, the proof reveals the structure of maximal $\sigma$-free subsets of $\{1, \ldots, n\}$ for sufficiently large $n$. For all integers $a, b, c$ satisfying $1 \leq a<b, \operatorname{gcd}(a, b)=1$, and $c \geq(a+b)^{3 / 2}$ the constructive part of the proof of Theorem 1 yields

$$
\bar{D}(\sigma) \geq(1-q) \frac{1-\beta+\alpha}{1-\beta},
$$

and we conjecture that this holds with equality, for $c$ sufficiently large.

For arithmetic reasons the lower bound for $c$ in Theorem 1 need not be optimal. In fact, Baltz et al. [1] investigated the precise structure of $\sigma$-free sets for $a=b=1$ and $c \geq 4$, and determined the value of $\bar{D}(\sigma)$ conjectured by Chung and Goldwasser [3]. By applying our method to the cases $c=4,5$, which are not directly covered by Theorem 1, we obtain an alternative proof of their density result.

Theorem 2 (Baltz, Hegarty, Knape, Larsson and Schoen [1]). Let $\sigma$ be defined by $x+y=c z$ with integer $c \geq 4$. Then

$$
\bar{D}(\sigma)=\frac{(c-2)\left(c^{2}-2\right)\left(c^{2}+2\right)}{c\left(c^{4}-2 c^{2}-4\right)} .
$$

The method of proof of Theorem 1 can also be used to determine the upper asymptotic density bound

$$
\bar{d}(\sigma)=\sup \{\bar{d}(A): A \subset \mathbb{N} \sigma \text {-free }\}
$$

for infinite $\sigma$-free sets of positive integers, where

$$
\bar{d}(A)=\limsup _{n \rightarrow \infty}\left(\frac{1}{n}|A \cap\{1, \ldots, n\}|\right)
$$

denotes the upper asymptotic density of the set $A \subseteq \mathbb{N}$. Since every finite subset of an infinite $\sigma$-free set is again $\sigma$-free, we have the general inequality $\bar{d}(\sigma) \leq \bar{D}(\sigma)$.

THEOREM 3. Let $\sigma$ be defined by (1.1) with positive integers $a, b, c$ satisfying $a \leq b, \operatorname{gcd}(a, b)=1$, and $c \geq \max \left\{2(a+b)^{2}-\operatorname{gcd}(a+b, c), a^{2}(b+1)\right\}$. 
Then

$$
\bar{d}(\sigma)=\frac{c^{2}-c(a+b)}{c^{2}-a(a+b)} .
$$

In particular, Theorem 3 covers also the case $a>1$. It follows from (1.2) and (1.4) that $\bar{d}(\sigma)=(1-q) /(1-\alpha)<\bar{D}(\sigma)$, under the assumptions of Theorem 3. Notice that the difference between $\bar{d}(\sigma)$ and $\bar{D}(\sigma)$ is very small. For instance, in the case $a=b=1$ and $c=6$ we obtain $\bar{d}(\sigma)=12 / 17=$ $0.70588235 \ldots$, while $\bar{D}(\sigma)=646 / 915=0.70601092 \ldots$.

Again, the lower bound for $c$ given in Theorem 3 need not be optimal. In fact, it was shown earlier (see [6] or [7]) that (1.4) holds for $c \geq 2 a b(a+b)$, but for $a>1$ the lower bound for $c$ given in Theorem 3 slightly improves the previous results. Special cases of infinite sum-free, $c$-sum-free or differencefree sets with $a=b=1$ and $c \neq 2$, or $a=-b=1$ were considered by Chung and Goldwasser [3], by Schoen [12], and by Euczak and Schoen [9]. For more than three variables see also Euczak and Schoen [8], and Deshouillers, Freiman, Sós and Temkin [5].

2. Construction. Throughout this paper we write $(s, t]$ for the intersection of the real interval $(s, t]$ with $\mathbb{N}$ and similarly $[s, t),(s, t)$, and $[s, t]$. We assume that $a, b, c \in \mathbb{N}$ satisfy $a \leq b$ and $a+b<c$, which gives $q=(a+b) / c<1$. If $x, y, z \in S:=(q m, m]$ with some $m \geq 1$ then $a S+b S$ is bounded above by $(a+b) m$, whereas the elements of $c S$ exceed $c q m$. Hence $a S+b S$ and $c S$ are disjoint sets so that $S$ is $\sigma$-free. We notice that, in general, $S$ is also an extremal segment, since for $m=c$ and $m^{\prime}=c-1$ the set $\{a+b, \ldots, c\}=[q m, m]=\left(q m^{\prime}, m^{\prime}+1\right]$ yields the solution $x=y=c$, $z=a+b$ to $a x+b y=c z$.

The idea of constructing extremal $\sigma$-free subsets $A$ of $[1, n]$ consists in taking the union of sufficiently distant disjoint segments of the above type. Suppose that for fixed $k \in \mathbb{N}$ a sequence of numbers $n_{1}=n>n_{2}>\cdots>n_{k}$ is chosen such that the $k \sigma$-free segments

$$
S_{j}=\left(q n_{j}, n_{j}\right] \quad(j=1, \ldots, k)
$$

are pairwise disjoint. In order to obtain a $\sigma$-free union $A=S_{1} \cup \cdots \cup S_{k}$, it suffices to arrange the largest element of the set $c\left(S_{j} \cup \cdots \cup S_{k}\right)$ below the smallest element of the set $a\left(S_{1} \cup \cdots \cup S_{j-1}\right)+b A$ for each $j=2, \ldots, k$. This yields

$$
n_{1}=n, \quad c n_{j} \leq a q n_{j-1}+b q n_{k} \quad(j=2, \ldots, k) .
$$

The number of elements of $A$ is

$$
|A|=(1-q)\left(n_{1}+\cdots+n_{k}\right)+r \quad \text { with } \quad|r| \leq k,
$$

which suggests that $|A|$ is maximal if all inequalities (2.2) are equalities. 
With $\alpha:=a q / c$ and $\beta:=b q / c$ this turns into

$$
n_{1}=n, \quad n_{j}=\alpha n_{j-1}+\beta n_{k} \quad(j=2, \ldots, k) .
$$

From this system of linear recurrence relations we obtain the upper segment bounds $n_{j}$ as functions of $n=n_{1}$ and $k$ : By multiplying the recurrence equations in (2.4) with $\alpha^{-j}$, summing over $j=2, \ldots, J$ and then replacing $J$ by $j$ we obtain

$$
n_{j}=\alpha^{j-1} n+\frac{1-\alpha^{j-1}}{1-\alpha} \beta n_{k} \quad(j=1, \ldots, k) .
$$

In particular, for $j=k$ it follows that

$$
n_{k}=\frac{(1-\alpha) \alpha^{k-1}}{1-(\alpha+\beta)+\alpha^{k-1} \beta} n
$$

and therefore

$$
n_{j}=\frac{1-(\alpha+\beta)+\alpha^{k-j} \beta}{1-(\alpha+\beta)+\alpha^{k-1} \beta} \alpha^{j-1} n \quad(j=1, \ldots, k) .
$$

Summation gives

$$
n_{1}+\cdots+n_{k}=\left(\frac{1-(\alpha+\beta)}{1-(\alpha+\beta)+\alpha^{k-1} \beta} \frac{1-\alpha^{k}}{1-\alpha}+\frac{k \alpha^{k-1} \beta}{1-(\alpha+\beta)+\alpha^{k-1} \beta}\right) n
$$

or

$$
n_{1}+\cdots+n_{k}=\frac{1+t_{k}}{1-\alpha} n
$$

where we have abbreviated

$$
t_{k}=\frac{(1-\alpha)((k-1) \beta-\alpha)}{1-(\alpha+\beta)+\alpha^{k-1} \beta} \alpha^{k-1} .
$$

By inserting into (2.3) we get

$$
|A|=\frac{1-q}{1-\alpha}\left(1+t_{k}\right) n+r \quad \text { with } \quad|r| \leq k
$$

which proves part (a) of the following lemma (for a weaker version see [7]).

LEMMA 1. With positive integers $a, b, c$ satisfying $a \leq b, \operatorname{gcd}(a, b)=1$, and $c \geq(a+b)^{3 / 2}$ let the system $\sigma$ be defined by (1.1). Further let $q=$ $(a+b) / c, \alpha=a q / c, \beta=b q / c$, and let the set $A \subseteq[1, n]$ be given by $A=$ $S_{k} \cup \cdots \cup S_{1}$ with the segments $S_{j}$ determined by (2.1) and (2.5). Then we have:

(a) The set $A$ is $\sigma$-free and has

$$
|A|=\frac{1-q}{1-\alpha}\left(1+t_{k}\right) n+r
$$

elements, where $|r| \leq k$ and $t_{k}$ is given by (2.6). 
(b) If $a=b$ then the maximal value of $|A|$ is attained for $k=3$, in which case

$$
|A|=(1-q) \frac{1-\alpha^{2}}{1-\alpha-\alpha^{2}} n+r \quad \text { and } \quad|r| \leq 3,
$$

whereas for $a<b$ it is attained for $k=2$, in which case

$$
|A|=(1-q) \frac{1-\beta+\alpha}{1-\beta} n+r \quad \text { and } \quad|r| \leq 2 .
$$

Proof. It remains to prove part (b). First we notice that (2.6) yields

$$
\begin{aligned}
& t_{1}=-\alpha<0, \\
& t_{2}=\frac{\alpha(\beta-\alpha)}{1-\beta} \begin{cases}=0 & \text { if } a=b, \\
>0 & \text { if } a<b,\end{cases} \\
& t_{3}=\frac{\alpha^{2}(2 \beta-\alpha)}{1-\beta(1+\alpha)}>0 .
\end{aligned}
$$

Next we show that the sequence $\left(t_{k}\right)$ is strictly decreasing for $k \geq k^{*}$ where $t_{k^{*}}$ is its first positive term. From (2.6) we get for $k \geq k^{*}$,

$$
\begin{aligned}
\vartheta_{k} & :=\frac{t_{k}}{t_{k+1}}=\frac{(k-1) \beta-\alpha}{k \beta-\alpha} \frac{1-(\alpha+\beta)+\alpha^{k} \beta}{1-(\alpha+\beta)+\alpha^{k-1} \beta} \frac{1}{\alpha} \\
& >\frac{\left(k^{*}-1\right) \beta-\alpha}{k^{*} \beta-\alpha} \frac{1-(\alpha+\beta)}{\alpha} \\
& =\frac{\left(k^{*}-1\right) b-a}{k^{*} b-a} \frac{c^{2}-(a+b)^{2}}{a(a+b)} .
\end{aligned}
$$

For $a=b$ and $k^{*}=3$ the first factor equals $1 / 2$, for $a<b$ and $k^{*}=2$ it equals $(b-a) /(2 b-a)$. In both cases it follows from $c \geq(a+b)^{3 / 2}$ that $\vartheta_{k}>1$ for $k \geq k^{*}$. Finally, by inserting the value of $t_{k^{*}}$ into (2.7) we obtain the asserted cardinality of $A$ in both cases for $k^{*}=3$ and for $k^{*}=2$, respectively.

3. Optimality. In order to prove that the construction in Section 2 is indeed optimal, we suppose that $A \subseteq\{1, \ldots, n\}$ is a given $\sigma$-free set. We are then going to show that the cardinality of this set $A$ cannot be much larger than in the construction above.

It follows from the construction in Section 2 that the segments $S_{j}$, whose upper bounds behave like the contracting geometric sequence $\left(n \alpha^{j-1}\right)$, are nonempty only for $j \ll \log n$. For a given $\sigma$-free set $A \subseteq\{1, \ldots, n\}$ with smallest element $s$ we adapt the above construction (notice that $\left[q n_{k}\right]=s-1$ ) and define the segments $T_{j}=\left(q n_{j}, n_{j}\right]$ by

$$
n_{1}=n, \quad n_{j}=\alpha n_{j-1}+b s / c \quad(j=2,3, \ldots) .
$$


The explicit solution to this linear recurrence relation is given by

$$
n_{j}=\alpha^{j-1} n+\frac{b s}{c} \frac{1-\alpha^{j-1}}{1-\alpha} \quad(j=1,2, \ldots) .
$$

From $\alpha \leq 1 / 4$ and $b<c(1-\alpha)$ we conclude that $n_{j}<s$ unless $j \ll \log n$. Let $T_{k}$ denote the last segment satisfying $s \leq \max T_{k}$. Then $k \ll \log n$ and by construction $U:=T_{1} \cup \cdots \cup T_{k}$ is $\sigma$-free.

The idea of the optimality proof for our construction in Section 2 consists in defining an almost injective map $\varphi: A \rightarrow U$, which means that we admit $\ll \log n$ exceptions from strict injectivity. It suffices to show that there is a map $\varphi: A \cap\left(n_{2}, n_{1}\right] \rightarrow T:=T_{1}$, which is injective apart from a number $\eta$ of exceptions depending at most on $a, b, c$. Then $A_{1}:=\left(A \cap\left[s, n_{2}\right]\right) \cup T_{1}$ is $\sigma$-free again, and $|A| \leq\left|A \cap\left[s, n_{2}\right]\right|+\left|T_{1}\right|+\eta$. By repeating this process with $A \cap\left[s, n_{2}\right]$ instead of $A=A \cap\left[s, n_{1}\right]$ and $n_{2}$ instead of $n_{1}$ we obtain $|A| \leq\left|A \cap\left[s, n_{3}\right]\right|+\left|T_{2}\right|+\left|T_{1}\right|+2 \eta$. After $k$ inductive steps we arrive at $|A| \leq\left|T_{k}\right|+\cdots+\left|T_{1}\right|+k \eta$. This means that the cardinality of $A$ is at most that of the $\sigma$-free union of $k$ disjoint segments $T_{j}$ for $j=1, \ldots, k \ll \log n$, apart from at most $k \eta \ll \log n$ exceptions. The assertion of Theorem 1 now follows from Lemma 1.

Following this strategy with $n_{1}=n$ and $n_{2}=a(a+b) n / c^{2}+b s / c$ we have to construct a mapping

$$
\varphi: A \cap\left(\frac{a(a+b) n+b c s}{c^{2}}, n\right] \rightarrow T=\left(\frac{(a+b) n}{c}, n\right]
$$

that is injective apart from $\eta \ll 1$ exceptions. We split the domain of $\varphi$ into three disjoint parts,

$$
\begin{aligned}
R & :=A \cap\left(\frac{(a+b) n}{c}, n\right], \\
L & :=A \cap\left(\frac{a(a+b) n+b c s}{c^{2}}, \frac{n}{c}\right], \\
M & :=A \cap\left(\frac{n}{c}, \frac{(a+b) n}{c}\right],
\end{aligned}
$$

and define three mappings $\varphi_{R}: R \rightarrow T, \varphi_{L}: L \rightarrow T$, and $\varphi_{M}: M \rightarrow T$ that determine $\varphi=\varphi_{R} \cup \varphi_{L} \cup \varphi_{M}$ as follows (see Figure 1).

We leave $R$ fixed under $\varphi_{R}$ so that

$$
\varphi_{R}: R \rightarrow T \text { with } \varphi_{R}(\xi):=\xi
$$

is injective and satisfies $\varphi_{R}(\xi) \in A$ for all $\xi \in R$. In order to define $\varphi_{L}$, we suppose here that $a=1$ and define

$$
\varphi_{L}: L \rightarrow T \text { by } \varphi_{L}(\xi):=\frac{c \xi-b s}{a} .
$$




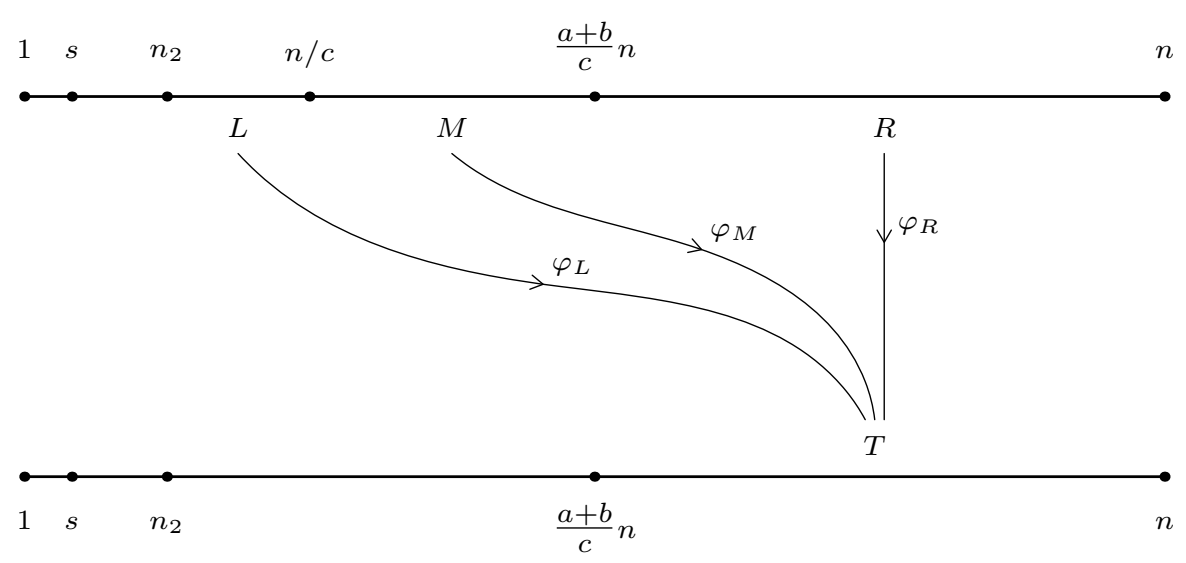

Fig. 1. The mapping $\varphi$ (not to scale)

Then $\varphi_{L}$ is injective and satisfies $\varphi_{L}(\xi) \notin A$ for all $\xi \in L$, since otherwise $x=\varphi_{L}(\xi), y=s$ and $z=\varphi_{R}(\xi)$ yield a solution in $A$ to $a x+b y=c z$, which is impossible. It follows from $\varphi_{R}(\xi) \in A$ and $\varphi_{L}(\xi) \notin A$ that $\varphi_{R} \cup \varphi_{L}$ is injective on $R \cup L$.

Since for $a>1$ the image set $\varphi_{L}(L)$ will have not only integer elements, the crucial step then consists in suitably modifying the definition of $\varphi_{L}$. This will be discussed in Section 5 .

The definition of $\varphi_{M}: M \rightarrow T$ is based on the integers in the open real interval with diameter $2(a+b)$ and center $c \xi /(a+b)$,

$$
K_{\xi}:=\left(\frac{c \xi}{a+b}-(a+b), \frac{c \xi}{a+b}+(a+b)\right)
$$

associated with $\xi \in M$. Let the subset $M^{\prime}$ of $M$ be defined by

$$
M^{\prime}=A \cap\left(\frac{n}{c}+(a+b), \frac{(a+b) n}{c}-(a+b)\right] .
$$

Evidently $\left|M \backslash M^{\prime}\right| \leq 2(a+b)$. We collect some useful properties of the sets $K_{\xi}$.

Lemma 2. For $c \geq 2(a+b)^{2}-\operatorname{gcd}(a+b, c)$ the following assertions hold:

(a) The sets $K_{\xi}$ for $\xi \in M$ are pairwise disjoint.

(b) For every $\xi \in M^{\prime}$ the set $K_{\xi}$ is a subset of $T$.

(c) For every $\xi \in M^{\prime}$ the set $K_{\xi} \backslash\left(\varphi_{R}(R) \cup \varphi_{L}(L)\right)$ is nonempty.

Proof. (a) It suffices to show that $K_{\xi}$ and $K_{\xi+1}$ have no common element for every $\xi \in \mathbb{N}$. We set $\delta=\operatorname{gcd}(a+b, c)$. From (3.5) we see that

$$
m_{\xi}^{\prime}:=\max K_{\xi}=\left[\frac{c \xi-\delta}{a+b}\right]+(a+b) \leq \frac{c \xi-\delta}{a+b}+(a+b)
$$


and

$$
m_{\xi+1}:=\min K_{\xi+1}=\left[\frac{c \xi+c}{a+b}\right]+1-(a+b)>\frac{c \xi+c}{a+b}-(a+b) .
$$

Now $c \geq 2(a+b)^{2}-\delta$ implies $m_{\xi+1}-m_{\xi}^{\prime}>0$ and hence $K_{\xi} \cap K_{\xi+1}=\emptyset$.

(b) From $\min M^{\prime}>n / c+(a+b)$ and $\min K_{\xi}>c \xi /(a+b)-(a+b)$ we see that the smallest element of all $K_{\xi}$ for $\xi \in M^{\prime}$ is larger than $n /(a+b)+$ $c-(a+b)$. Hence we have to verify that

$$
\frac{n}{a+b}+c-(a+b)>\frac{a+b}{c} n
$$

or, equivalently,

$$
n\left(c-(a+b)^{2}\right)>-(a+b) c(c-(a+b)),
$$

which holds trivially.

Since $\max M^{\prime} \leq(a+b) n / c-(a+b)$ and $\max K_{\xi}<c \xi /(a+b)+(a+b)$, the largest element of all $K_{\xi}$ for $\xi \in M^{\prime}$ is at most $n-c+(a+b)<n$.

(c) The cardinality of the set $K_{\xi}$ equals $2(a+b)$ if $c \xi \not \equiv 0 \bmod (a+b)$, and $2(a+b)-1$ if $c \xi \equiv 0 \bmod (a+b)$.

Consider any pair of not necessarily distinct elements $\frac{c \xi-\lambda}{a+b}, \frac{c \xi+\mu}{a+b} \in K_{\xi}$ such that $a \lambda=b \mu$. They cannot both belong to $\varphi_{R}(R)$, since otherwise the equation $a x+b y=c z$ is solved in $A$ by $x=(c \xi-\lambda) /(a+b)$, $y=(c \xi+\mu) /(a+b)$ and $z=\xi$, which is impossible. The number of such pairs of elements in $K_{\xi}$ equals the number of solutions to $a \lambda=b \mu$ in the range $-(a+b)^{2}<\lambda, \mu<(a+b)^{2}$ such that $a+b$ divides both $c \xi-\lambda$ and $c \xi+\mu$. Since $a$ and $b$ are coprime, the solutions to $a \lambda=$ $b \mu$ in the above range are $\lambda=t b$ and $\mu=t a$ with integers $t$ satisfying $-(a+b)^{2} / b<t<(a+b)^{2} / b$. Only those $t$ are admissible for which $t b \equiv c \xi \bmod (a+b)$ and $t a \equiv-c \xi \bmod (a+b)$. Since these congruences are equivalent and $b$ is coprime to $a+b$, there exists precisely one fundamental solution $t_{0} \in\{0, \ldots, a+b-1\}$. In the above range for $t$ we thus obtain two different parameters $t_{0}$ and $t_{0}-(a+b)$ that yield two distinct pairs of elements $\frac{c \xi-\lambda}{a+b}, \frac{c \xi+\mu}{a+b} \in K_{\xi}$ such that $a \lambda=b \mu$. Therefore $\left|K_{\xi} \backslash \varphi_{R}(R)\right| \geq 2$.

Finally we notice that successive elements of $\varphi_{L}(L)$ have at least the distance $c$ so that at most one element of $K_{\xi}$ belongs to $\varphi_{L}(L)$. Therefore the difference set $K_{\xi} \backslash\left(\varphi_{R}(R) \cup \varphi_{L}(L)\right)$ is nonempty for every $\xi \in M^{\prime}$.

By Lemma 2 we may define

$$
\varphi_{M}: M^{\prime} \rightarrow T \quad \text { by } \quad \varphi_{M}(\xi):=\max K_{\xi} \backslash\left(\varphi_{R}(R) \cup \varphi_{L}(L)\right)
$$

and extend $\varphi_{M}$ from $M^{\prime}$ to $M$ by $\varphi_{M}(\xi)=\max T$ for $\xi \in M \backslash M^{\prime}$, say. Then $\varphi_{M}$ is injective on $M^{\prime}$ and $\varphi_{L} \cup \varphi_{R} \cup \varphi_{M}: L \cup R \cup M \rightarrow T$ is injective by construction, apart from $\eta \leq 2(a+b)$ exceptions. According to our previously 
explained strategy, Theorem 1 is proved for $\sigma$ defined by $a x+b y=c z$ with integers $b \geq a=1$ and $c \geq 2(a+b)^{2}-\operatorname{gcd}(a+b, c)$.

4. Proof of Theorem 2. For $a=b=1$ and $c \geq 6$ Theorem 2 is contained in Theorem 1 . Therefore it suffices to study the two cases $c=4,5$ with $a=b=1$ separately.

Due to overlapping segments $K_{\xi}$ in the remaining cases $c=4$ and $c=5$, we have to refine the investigation of the mapping $\varphi_{M}: M \rightarrow T$ and start with modifying the definition of $K_{\xi}$. For convenience we set $\xi_{s}:=[n / c]+1$ and $\xi_{l}:=[2 n / c]$.

For $c=4$ and $\xi \in M$ we define

$$
\begin{array}{ll}
K_{\xi}:=\{2 \xi\} & \text { if } 2 \xi \notin \varphi_{L}(L), \\
K_{\xi}:=\{2 \xi-1,2 \xi, 2 \xi+1\} & \text { if } 2 \xi \in \varphi_{L}(L)
\end{array}
$$

and note the following properties of these sets.

Lemma 3. For $a=b=1$ and $c=4$ let $M^{\prime}:=M \backslash\left\{\xi_{s}, \xi_{l}\right\}$. Then, with the sets $K_{\xi}$ defined in (4.1) and (4.2), the assertions of Lemma 2 remain true.

Proof. (a) We observe that successive elements of $\varphi_{L}(L)$ have at least the distance 4 . Hence, for $\xi, \xi+1 \in M$ one of the sets $K_{\xi}$ and $K_{\xi+1}$ is of type (4.1), which gives the assertion.

(b) In order to guarantee $K_{\xi} \subset T$ for all $\xi \in M^{\prime}$, in addition to the assumptions of Lemma $2(\mathrm{~b})$ the element $\xi_{s}=[n / 4]+1$ has to be excluded from the set $M$.

(c) For $\xi \in M^{\prime}$ we have $2 \xi \notin \varphi_{R}(R)$, since otherwise $x=y=2 \xi$ and $z=\xi$ yield a solution to $x+y=4 z$, a contradiction. In the case (4.1) the assertion follows. In the case (4.2) we notice that $2 \xi-1$ and $2 \xi+1$ cannot both belong to $\varphi_{R}(R)$. By the distance condition none of them is in $\varphi_{L}(L)$. This gives the assertion.

Due to Lemma 3 we may now define $\varphi_{M}: M^{\prime} \rightarrow T$ by (3.6) and extend the domain of $\varphi_{M}$ to $M$ by $\varphi_{M}\left(\xi_{s}\right)=\min T$ and $\varphi_{M}\left(\xi_{l}\right)=\max T$, say. Then $\varphi_{M}: M \rightarrow T$ is well defined and, by construction, $\varphi=\varphi_{L} \cup \varphi_{R} \cup \varphi_{M}$ maps $L \cup R \cup M$ injectively into $T$, apart from $\eta \leq 2$ exceptions.

In the case $a=b=1, c=5$ we set $\xi^{\prime}:=5 \xi / 2$ for $\xi \in M \cap 2 \mathbb{N}$ and consider

$$
\begin{aligned}
K_{\xi} & :=\left\{\xi^{\prime}\right\} & & \text { if } \xi^{\prime} \notin \varphi_{L}(L), \\
K_{\xi} & :=\left\{\xi^{\prime}-1, \xi^{\prime}, \xi^{\prime}+1\right\} & & \text { if } \xi^{\prime} \in \varphi_{L}(L), \\
K_{\xi \pm 1} & :=\left\{\xi^{\prime} \pm 1, \xi^{\prime} \pm 2, \xi^{\prime} \pm 3, \xi^{\prime} \pm 4\right\} & & \text { if } \xi^{\prime} \notin \varphi_{L}(L), \\
K_{\xi \pm 1} & :=\left\{\xi^{\prime} \pm 2, \xi^{\prime} \pm 3\right\} & & \text { if } \xi^{\prime} \in \varphi_{L}(L) .
\end{aligned}
$$


Lemma 4. For $a=b=1$ and $c=5$ let $M^{\prime}:=M \backslash\left\{\xi_{l}\right\}$. Then, with the sets $K_{\xi}$ and $K_{\xi+1}$ defined in equations (4.3) to (4.6), the assertions of Lemma 2 remain true.

Proof. Notice that successive segments $K_{\xi}, K_{\xi+1}$ or $K_{\xi-1}$ are either of the types (4.3) and (4.5), or of the types (4.4) and (4.6). The verification of assertions (a) to (c) now follows the previous lines and uses the fact that the distance of consecutive elements of $\varphi_{L}(L)$ is at least 5 .

By Lemma 4 the definition of $\varphi_{M}: M^{\prime} \rightarrow T$ follows (3.6) and is extended to $M$ by $\varphi_{M}\left(\xi_{l}\right)=\max T$, say. Again $\varphi_{M}: M \rightarrow T$ is well defined and by construction $\varphi=\varphi_{L} \cup \varphi_{R} \cup \varphi_{M}: L \cup R \cup M \rightarrow T$ is injective, apart from $\eta \leq 1$ exceptions.

This completes the proof of Theorem 2 for $a=b=1$ and $c \geq 4$.

5. The case $a>1$. In order to carry through the optimality proof of our construction also in the case $a>1$ via the methods of Section 3, we have to modify the definition of $\varphi_{L}: L \rightarrow T$ in equation (3.4),

$$
\varphi_{L}(\xi)=\frac{c \xi-b s}{a} .
$$

Thus we associate to each $\xi \in L$ a number $s(\xi) \in A$, as close to $s$ as possible, such that $c \xi-b s(\xi) \equiv 0 \bmod a$, and alter the above definition to

$$
\varphi_{L}(\xi):=\frac{c \xi-b s(\xi)}{a} .
$$

Since $a$ and $b$ are coprime, we note that for any given $\xi \in L$ the preceding congruence has a unique solution $s(\xi) \in \mathbb{Z} / a \mathbb{Z}$. Hence we have to show that every residue class modulo $a$ contains elements of $A$.

A vital point is the injectivity of the mapping $\varphi_{L}$. Let $b^{\prime}$ and $c^{\prime}$ be defined by $b=b^{\prime} \operatorname{gcd}(b, c)$ and $c=c^{\prime} \operatorname{gcd}(b, c)$. Suppose that $\varphi_{L}(\xi)=\varphi_{L}\left(\xi^{\prime}\right)$. Then $c \xi-b s(\xi)=c \xi^{\prime}-b s\left(\xi^{\prime}\right)$ or, equivalently, $c\left(\xi-\xi^{\prime}\right)=b\left(s(\xi)-s\left(\xi^{\prime}\right)\right)$ shows that $s(\xi)$ and $s\left(\xi^{\prime}\right)$ necessarily belong to the same residue class modulo $c^{\prime}$. Hence we have indeed to ensure that there exists a complete residue system $\Gamma \subseteq A$ modulo $a$ of distinct representatives modulo $c^{\prime}$. Finally, we have to choose the elements of $\Gamma$ as small as possible in order to obtain a small cardinality of the difference set $\varphi_{L}(L) \backslash T$, which characterizes the exceptions that have to be made from the injectivity of $\varphi_{L}: L \rightarrow T$.

We may assume that $A$ has more than $(1-q) n$ elements, since otherwise $A$ can trivially be mapped injectively into $T$. We claim that no subset $A_{\varrho}:=$ $\{x \in A: x \equiv \varrho \bmod a\}, \varrho=0, \ldots, a-1$, is empty. This follows from $n-q n<|A| \leq(a-1)[n / a]+\left|A_{\varrho}\right|$. In fact, $\left|A_{\varrho}\right|>(1 / a-q) n$. Hence, for any given $\xi \in L$, the congruence $b s(\xi) \equiv c \xi \bmod a$ has many solutions $s(\xi) \in A$. 
Lemma 5. Let $A \subseteq\{1, \ldots, n\}$ be $\sigma$-free, where $\sigma$ is defined by ax + by $=c z$ with integers $a, b, c$ satisfying $1 \leq a \leq b, \operatorname{gcd}(a, b)=1$, and $c \geq$ $a^{2}(b+1)$, and let $c=c^{\prime} \operatorname{gcd}(b, c)$. If

$$
|A|>(1-q) n \geq a
$$

then there exists a complete residue system $\Gamma \subseteq A$ modulo a of numbers that are distinct modulo $c^{\prime}$.

Proof. It suffices to show that every subset $A_{\varrho}=\{x \in A: x \equiv \varrho \bmod a\}$ contains at least $a$ numbers $s_{\varrho}^{\prime}$ that are distinct modulo $c^{\prime}$. Since $c^{\prime} \geq c / b>a$ and $\operatorname{gcd}(a, b)=1$, the numbers $s_{\varrho}$ then appear as an appropriate selection from the numbers $s_{\varrho}^{\prime}$. Suppose, to the contrary, that one of the sets $A_{\varrho}$, say $A_{\varrho^{\prime}}$, contains fewer than $a$ distinct elements modulo $c^{\prime}$. Then $\left|A_{\varrho^{\prime}}\right| \leq$ $(a-1) n / c^{\prime}$. Combining this with $(1-q) n<|A| \leq(1-1 / a) n+\left|A_{\varrho^{\prime}}\right|$ and $b c^{\prime} \geq c$ we obtain

$$
1-q<1-\frac{1}{a}+\frac{(a-1) b}{c}
$$

which yields $c<a^{2}(b+1)$, a contradiction.

Lemma 5 shows that for every $\varrho \in\{0, \ldots, a-1\}$ there exists a smallest solution $s_{\varrho} \in A$ to $b s_{\varrho} \equiv c \varrho \bmod a$ such that the collection $\Gamma:=\left\{s_{0}, \ldots, s_{a-1}\right\}$ consists of distinct residue classes modulo $c^{\prime}$. This $s_{\varrho}$ then serves as $s(\xi)$ for every element $\xi \in L \cap A_{\varrho}$, which guarantees injectivity of $\varphi_{L}$ on $L \cap A_{\varrho}$. Clearly we have $s=\min \Gamma$ and it remains to estimate $\max \Gamma-s$. Any inequality of the type

$$
\max \Gamma-s \leq E(n)
$$

with a function $E(n) \ll n^{1-\varepsilon}$ and some fixed $\varepsilon>0$, instead of an upper bound independent from $n$, would suffice to determine $\bar{D}(\sigma)$. We have to leave this as an open problem.

6. Infinite $\sigma$-free sets as limiting case. Lemma 5 may be used to easily derive a new proof for the value of the upper asymptotic bound $\bar{d}(\sigma)$ defined in (1.3) for infinite $\sigma$-free sets of positive integers.

Let again $a, b, c$ be positive integers satisfying $a \leq b$ and $c>a+b$. As in Section 2 let $q=(a+b) / c$ and $\alpha=a(a+b) / c^{2}$. For $k \in \mathbb{N}$ we set $m_{k}:=\alpha^{1-k}$. From the general construction principles for $\sigma$-free sets we see that the set

$$
A=\bigcup\left\{\left(q m_{k}, m_{k}\right]: k=1,2, \ldots\right\}
$$

is $\sigma$-free with $\min A=1$. The upper asymptotic density $\bar{d}(A)$ of the set $A$ is then given by

$$
\bar{d}(A)=(1-q) \lim _{k \rightarrow \infty} \frac{m_{1}+\cdots+m_{k}}{m_{k}}=\frac{1-q}{1-\alpha} .
$$


Notice that this result (see [6], [7]) may be considered the limiting case of the construction of finite $\sigma$-free sets $A$, which is obtained from (2.7) by taking $k \asymp \log n$, dividing by $n$ and then taking the limit $n \rightarrow \infty$.

By inserting the values of $q$ and $\alpha$ the above construction shows that

$$
\bar{d}(\sigma) \geq \frac{c^{2}-c(a+b)}{c^{2}-a(a+b)} .
$$

In order to prove that this bound is optimal for all positive integers $a, b, c$ satisfying $a \leq b, c \geq \max \left\{2(a+b)^{2}-\operatorname{gcd}(a+b, c), a^{2}(b+1)\right\}$, and $\operatorname{gcd}(a, b)=1$, we may suppose that $A \subset \mathbb{N}$ is $\sigma$-free and has the upper asymptotic density $\bar{d}(A)>1-q$. Then there exists a strictly increasing sequence of numbers $n^{*} \in \mathbb{N}$ such that the truncated set $A_{n^{*}}:=A \cap\left\{1, \ldots, n^{*}\right\}$ has the cardinality

$$
\left|A_{n^{*}}\right|>(1-q) n^{*} \text {. }
$$

For $n^{*} \geq a /(1-q)$ Lemma 5 yields the existence of a complete residue system $\Gamma \subseteq A_{n^{*}}$ modulo $a$ of elements of $A$ that are distinct modulo $c^{\prime}$. Notice that the choice of $\Gamma$ does no longer depend on $n^{*}$, since the subsets $A_{n}$ of $A$ are ordered by inclusion. Let $\gamma:=\max \Gamma$. Then, with $A$ replaced by $A_{n^{*}} \cap\left(\gamma, n^{*}\right]$, there is an injective mapping $\varphi: R \cup L \cup M \rightarrow T$ except for a number $\eta$ of exceptions which we may estimate similarly to Section 3 in the following way: Since $\varphi_{R}$ is the identity mapping on $R \subseteq T$, there is no exception. Since for every $\xi \in L$ there is a unique $s(\xi) \in \Gamma$ such that $c \xi-b s(\xi) \equiv 0 \bmod a$, the mapping $\varphi_{L}: L \rightarrow T$ is well defined by (5.1) if $(c \xi-b s(\xi)) / a \in T$ and by $\varphi_{L}(\xi)=\min T$ if $(c \xi-b s(\xi)) / a \notin T$. Here exceptions from injectivity are only caused by those $\xi \in L$ satisfying

$$
\frac{c \xi-b s(\xi)}{a}=\frac{c \xi-b s}{a}-\frac{b}{a}(s(\xi)-s) \leq \frac{a+b}{c} n^{*}
$$

so that their number is bounded by $b \gamma / a$. Further, the mapping $\varphi_{M}$ defined by (3.6) is injective apart from at most $2(a+b)$ exceptions. Hence

$$
\eta \leq 2(a+b)+\frac{b}{a} \gamma
$$

Now we apply the strategy described at the beginning of Section 3 . The number of successive mappings that are necessary to map $A_{n^{*}}$ into a $\sigma$-free union of segments of type $T$ and $A \cap[1, \gamma]$ is of the order $k \asymp \log n^{*}$, each with at most $\eta$ exceptions from injectivity. Hence we obtain

$$
\left|A_{n^{*}}\right| \leq \frac{1-q}{1-\alpha}\left(1+t_{k}\right) n^{*}+k \eta
$$

Since $t_{k}$ tends to 0 as $n^{*}$ tends to $\infty$, Theorem 3 follows.

Notice that this proof is not restricted to $a=1$ and may be considered as the limiting case of the analogous result for finite $\sigma$-free sets proved only for $a=1$. 
Acknowledgements. The second author is indebted to the Department of Mathematics and Statistics at Dalhousie University in Halifax for excellent working conditions during his stay in August and September 2004.

\section{References}

[1] A. Baltz, P. Hegarty, J. Knape, U. Larsson and T. Schoen, The structure of maximum subsets of $\{1, \ldots, n\}$ with no solutions to $a+b=k c$, Electron. J. Combin. 12 (2005), no. 19.

[2] F. R. K. Chung, P. Erdôs and R. Graham, On sparse sets hitting linear forms, in: M. A. Bennett et al. (eds.), Number Theory for the Millennium, Vol. I (Urbana, IL, 2000), A K Peters, Natick, MA, 2002, 257-272.

[3] F. R. K. Chung and J. L. Goldwasser, Maximum subsets of $(0,1]$ with no solutions to $x+y=k z$, Electron. J. Combin. 3 (1996), no. 1 .

[4] - - - Integer sets containing no solutions to $x+y=3 z$, in: R. L. Graham and J. Nešetřil (eds.), The Mathematics of Paul Erdôs, Springer, Berlin, 1997, 218-227.

[5] J.-M. Deshouillers, G. A. Freiman, V. Sós and M. Temkin, On the structure of sumfree sets, 2, in: J.-M. Deshouillers et al. (eds.), Structure Theory of Set Addition, Astérisque 258 (1999), 149-161.

[6] L. Lucht, Dichteschranken für die Lösbarkeit gewisser linearer Gleichungen, J. Reine Angew. Math. 285 (1976), 209-217.

[7] -, Extremal pattern-free sets of positive integers, Ann. Univ. Sci. Budapest. Sect. Comput. 22 (2003), 253-268.

[8] T. Euczak and T. Schoen, On the infinite sum-free sets of natural numbers, J. Number Theory 66 (1997), 211-224.

[9] —, - Solution-free sets for linear equations, ibid. 102 (2003), 11-22.

[10] I. Z. Ruzsa, Solving a linear equation in a set of integers I, Acta Arith. 65 (1993), 259-282.

[11] —, Solving a linear equation in a set of integers II, ibid. 72 (1995), 385-397.

[12] T. Schoen, On sets of natural numbers without solution to a noninvariant linear equation, ibid. 93 (2000), 149-155.

Department of Mathematics and Statistics

Dalhousie University

Halifax, Nova Scotia B3H 3J5, Canada

E-mail: dilcher@mathstat.dal.ca
Institut für Mathematik Technische Universität Clausthal 38678 Clausthal-Zellerfeld, Germany E-mail: lucht@math.tu-clausthal.de 